\title{
The Internalization of Islamic Creed among the Muslim Adolescents in Malaysia
}

\author{
Norsaleha Mohd. Salleh ${ }^{1}$ \\ Ab. Halim Tamuri ${ }^{1}$ \\ Abur Hamdi Usman ${ }^{1}$ \\ Noor Hafizah Mohd. Haridi ${ }^{1}$ \\ Mahfuzah Mohammed Zabidi² \\ ${ }^{1}$ Department of Usuluddin, International Islamic University College Selangor, Malaysia \\ Corresponding author: norsaleha@kuis.edu.my \\ ${ }^{2}$ Academy of Contemporary Islamic Studies, UITM Shah Alam, Selangor, Malaysia
}

Doi:10.5901/mjss.2015.v6n6s4p18

\section{Abstract}

This study aimed to identify the stage of the internalization of akidah (Islamic creed) among the Muslim adolescents in Malaysia. The research conceptual framework was based on the principle of akidah formulated based on the Quran, the prophetic tradition and the model of akidah internalization. The framework was linked with the National Education and Islamic Education Philosophy which emphasize on the soul and spiritual peacefulness. The research utilized survey and interview as the data collection methods. All of the data collection instruments were modified by the researchers and validated by panel of experts. The Cronbach Alpha reliability test of the instruments showed a high score of $(>0.8)$. The research utilized the strata random sampling technique. A total of 1520 form four students from 24 Secondary Schools in Malaysia were selected in this study. The data was analyzed in descriptive using mean and standard deviation. Generally, the descriptive analysis findings showed a relatively high level of internalization of akidah, among the Muslim adolescents. In conclusion, the akidah of Muslims adolescents in Malaysia is seen well and under control. There is a good number of adolescents who are practicing akidah and the teachings of Islam. However this belief should be engrained at early age as al-Ghazali's theory education asserted the importance of the appreciation of akidah to be engrained since childhood. The findings of the study are useful to policy makers in the attempt to strengthen the internalization of akidah among the Muslim adolescents which is in line with the National Education mission and vision.

Keywords: Akidah; Adolescent; Malaysia; Education; al-Ghazali's Theory

\section{Introduction}

The Muslim adolescents are the invaluable asset of the nation and future leaders. And as a member of the society, the concerns and worries towards the decay of the internalization of their creed are inevitable. The society, as much as possible would like to see that all adolescents are aware of the reality including the responsibility and trust that they are carrying on their shoulders. The low statistic and minute percentage are not excuses for educators including parents to be in the state of contentment as that small numbers might spark even bigger problems later on. If the existence of that number is not dealt properly, it eventually will become a major threat to the Muslims one day.

Internalization can be summarized as the integration of attitudes, beliefs, values and such into one's personality. It is a form of conformity; in which the internalization of values or belief is the most permanent and deeply rooted impact of social influence. This is due to the embedded motivation within one's heart which seeks to internalize values or certain beliefs; and is related to the desire towards the truth. It is also said to be a kind of learning and subject to the laws of learning (Langgulung 1987). If we fathom the term internalization, it expresses the desire to observe something completely and to hold on to the truth steadfastly.

In the context of internalization of Islamic creed (akidah), al-Ghazali (n.d) believed one needs to undergo the conditioning process and exercises that will facilitate the emergence of obedience which later develops into habits. AlGhazali had outlined few preliminary steps to ensure children and adolescents could observe akidah rightfully and authentically. The initial steps were memorizing, understanding and trusting what they have learned about the knowledge 
of akidah. In addition, in ensuring akidah to be deeply rooted and solidly embedded in their hearts, each child and adolescent needs guidance when they are performing the practice and obligations deemed upon Muslims.

\section{Problem Statement}

The problem revolving the internalization of akidah among Muslim adolescents was discussed by Ibrahim (2004) by illustrating it through the involvement of adolescents in activities that would destruct akidah such as black metal. Black metal was linked to the wild, brutal, inverted and Satan-worshipping music genre that is capable of diverting akidah and leading adolescents into serious moral decay (Hassan, 2011 and Tee, 2009). The group commonly engaged in activities that insult the akidah of Islam such as offending the Prophet Muhammad (pbuh) (Hassan, 2011); and treading and burning the Qur'an. Besides that, black metal members were also involved in other negative activities such as free sex, intoxicant, drug addiction, psychoactive drugs abuse and practicing lifestyle that was against societal norms (Ibrahim, 2004 and Wan Othman, 2003).

The research conducted by Ibrahim (2004) in Sungai Petani, Kedah on 350 adolescents who were involved in social problems including black metal, discovered that 159 or $58.3 \%$ of the adolescents involved in black metal were due to the lack of religious education. This indicates the importance of instilling religious education especially akidah of Islam at a very tender age. According to al-Ghazali (2005), a close bond with Allah SWT will cultivate an emotionally and spiritually smart individual. HAMKA (2003) and Ibrahim (1996) have also acknowledged the view, expressing that the right akidah is able to lead and guide humans towards developing and cultivating a sound personality which later will be reflected thru being truly emotionally and spiritually smart.

There are several contributing reasons behind the influence of black metal among adolescents such as peer influence, desire to have fun and try new thing, interest in loud music; and inclination towards free mingling between genders (Wan Othman, 2003). According to al-Ghazali (n.d) and Ulwan (1998), naturally, human comes with the attribute of copying in which one could obtain both good and bad traits through his or her association with other people. If one associates with a kind and pious person, he or she would eventually obtain certain good traits from such friend. Likewise, if one associates with an unkind and corrupted person, the corrupted traits of such friend would also be able to influence one's behavior and attitude.

Ibn Khaldun (2009) asserted that the goodness in an individual must originate from within him or herself and from his or her awareness towards family and environmental factors; and the role of both in forming human characters. Therefore, it is necessary for adolescents to master knowledge especially the one related to religious values such as akidah, akhlak (character) and ibadah (worship). Today, the education system demands mastery in knowledge. Nevertheless, what is more important is to what extent students would be able to translate the knowledge they have learnt into their everyday lives. How far the knowledge is able to form the way of thinking, formulating opinions, expressing emotions and feelings; and acting and interacting with others (al-Ghazälī, n.d; Shaleh \& Wahab 2005; Manap et al. 2004 and Langgulung, 1987).

The problem of akidah among those who were involved in black metal negative culture was not only contributing to social problem but it was also contributing to the increase of emotional disturbance among them (Bedu et al. 2008; Ibrahim, 2004). Emotional disturbance takes place when one encounters certain circumstance that bothers the heart and emotion; it is a result from the pressure contributed by the surrounding people. At the same time, he or she is unsuccessful in controlling his or her own emotion, behavior, cognitive, physiology and later fail to develop good human relationship with others (Haque, 2001; Kartono1982 and Daradjat 1970). The Ministry of Health Malaysia (2008) report regarding a research conducted by The Third National Health and Morbidity Survey (NHMS III) in 2006 on children and adolescents who suffer from mental health in Malaysia showed a rather high increase. The emotional disturbance problem among Malay children and adolescents had increased at about 8.5\%, from $11.9 \%$ in 1996 to $20.4 \%$ in 2006 .

The increase is worrying as school students or adolescents are the nation's most important asset. In fact, the statistic illustrated a chronic situation among the present day adolescents especially those among the Muslims. Adolescents who suffers from such emotional problem will not only harm themselves, but they would also cause harm to the safety and security of others such as by committing murder, rape, molestation and by inflicting injury (Desa, 2004). According to al-Qarādawī (2005) and Sābiq (2003), the main reason for one to suffer from litany and distress to the extent leading towards emotional disturbance and inclination towards suicide was that, they have drifted away from religion and their relationship with Allah is extremely fragile. Hence, the adolescents who had a highly comprehension on their creed could affect of social environment. 


\section{Research Methodology}

The methodology of this research is as follows:

\subsection{Sample and data sampling method}

The design of this research was a survey method involving descriptive data analysis which has enabled the researcher to make generalization over the population based on the selected sample. It utilized a set of akidah Internalization Scale questionnaire. The data collection was carried out using quantitative method involving the questionnaire distribution to 1520 samples among form 4 students from 24 National Secondary Schools in Malaysia which were selected based on stratified random sampling method.

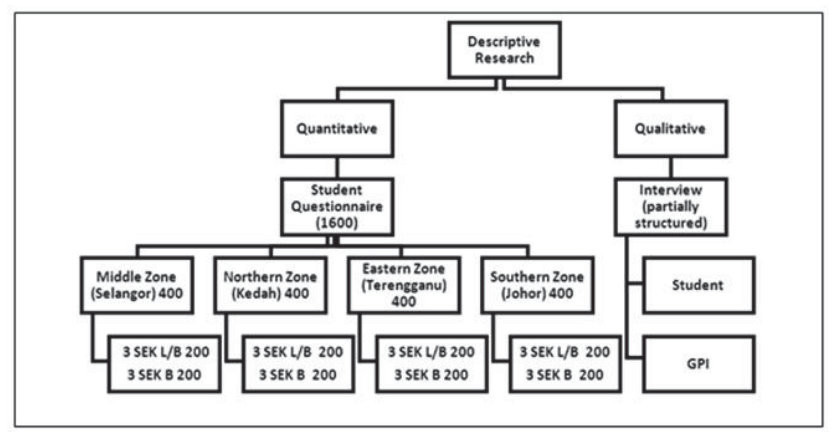

Figure 1: Research design

\subsection{Research Instrument}

The modification of the research instrument was done by referring to the akidah Learning Model by Ismail (2009), Islamic Studies Internalization Scale by Mahmood (2006) and Internalization of Akidah Model by Sābiq (2003). The modification was made to suit the Islamic culture in Malaysia.

\subsection{Instrument Validity and Reliability}

\subsubsection{Content validity and face validity}

The content of all modified data collection instrument was validated by a reference panel consisting of nine experts. Based upon the discussion with the experts, the irrelevant items to the construct were removed. Only $100 \%$ constructconformed items were selected.

\subsubsection{Pilot study and instrument reliability value}

To evaluate the reliability of the instrument, a pilot study was carried out on 100 form 4 students. The level of Alpha Cronbach reliability value yielded from all instrument was high $(>0.8)$, which normally indicates a high reliability level and can be accepted as one of the instrument (Bryman \& Cramer 2011; Abu \& Tasir, 2001).

\subsubsection{Normality test}

Normality test was also conducted on sample who took Islamic Studies paper in Penilaian Menengah Rendah (PMR) ${ }^{1}$ 2012. The Kolmogorov-Smirnov normality test revealed a significant value of 0.000 . Palant (2011) suggested the value can be accepted as normal distribution due the large number of research sample of 1520 students.

1 Lower Secondary Evaluation (LSE), a public examination for form three secondary school students. 
Table 1. Normality Test

\begin{tabular}{llccc} 
& Variable & \multicolumn{3}{c}{ Kolmogorov-Smirnova } \\
\cline { 2 - 5 } & Statistic & Df & Sig. \\
\cline { 2 - 5 } Internalization of Akidah & 0.073 & 1520 & 0.000 \\
\hline
\end{tabular}

a Liliefors Significance Correction

\section{Research Findings}

The internalization of akidah is referred to three main domains, namely comprehension, feelings and practice. These were followed by six sub-domains which consisted of the six pillars of iman (conviction of faith) which are learnt in the akidah lesson as part of the $\mathrm{KBSM}^{2}$ secondary school Islamic education syllabus. Generally, the mean score analysis for all domains as shown in Table 2 illustrated the trend in the internalization of akidah among the respondents showed high scores in the behavioral interpretation.

Table 2. Mean Score for Student Internalization of Akidah

\begin{tabular}{lccc}
\hline Main Domain & Mean & Standard Deviation & Interpretation \\
\hline Feelings & 4.64 & 0.31 & High \\
Comprehension & 4.58 & 0.31 & High \\
Practice & 4.09 & 0.42 & High \\
\hline
\end{tabular}

In Table 2, the three main domains of internalization of akidah obtained high scores. The mean score for comprehension domain was $=4.58(p=0.31)$, the mean for feelings domain was $=4.64(p=0.31)$ and the mean for practice domain was $=4.09(p=0.42)$. This showed the level of internalization of akidah among all respondents was very high. Nevertheless, the mean score for practice domain (mean $=4.09, p=0.42$ ) was slightly low compared to the mean score obtained for comprehension domain (mean $=4.58, p=0.31$ ) and the mean score for feelings domain (mean $=4.64, p=0.31$ ).

Hence, in order to elaborate in further details each domain and sub domain of internalization of akidah of Muslim adolescents in Malaysia; descriptive analysis tools such as frequency, percentage, mean score and standard deviation were used. Meanwhile, the respondents' responses were obtained using the Likert Scale: Strongly Disagree = SD, Disagree $=\mathrm{D}$, Less Agree $=\mathrm{LA}$, Agree $=\mathrm{A}$ and Strongly Agree $=\mathrm{SA}$.

\section{Internalization of Akidah: Overall Analysis}

The descriptive analysis distribution of internalization of akidah among respondents is shown in Table 3 as follows. The scores for akidah items that were stated in negative form $\left(^{*}\right)$ were recoded prior to the analysis in order to capture the actual level of internalization of akidah mean score.

Table 3. Respondents Internalization of Akidah

\begin{tabular}{|c|c|c|c|c|c|c|c|c|}
\hline \multirow{2}{*}{ Item Code } & \multirow{2}{*}{ Item } & \multicolumn{5}{|c|}{ Frequency \& Percentage $(\mathrm{N}=1520)$} & \multicolumn{2}{|c|}{ Mean \& SD } \\
\hline & & STS & TS & KS & $\mathrm{S}$ & SS & & \\
\hline B01 & Convinced in the existence of God & $\begin{array}{c}6 \\
(0.4)\end{array}$ & $\begin{array}{c}4 \\
(0.3)\end{array}$ & $\begin{array}{c}33 \\
(2.1)\end{array}$ & $\begin{array}{c}256 \\
(16.9)\end{array}$ & $\begin{array}{c}1221 \\
(80.3)\end{array}$ & 4.78 & 0.54 \\
\hline $\mathrm{B} 02 *^{*}$ & Repentance after sinning is unnecessary & $\begin{array}{c}24 \\
(1.6)\end{array}$ & $\begin{array}{c}9 \\
(0.6)\end{array}$ & $\begin{array}{c}18 \\
(6.7)\end{array}$ & $\begin{array}{l}102 \\
(6.7)\end{array}$ & $\begin{array}{l}1367 \\
(89.9)\end{array}$ & 4.83 & 0.63 \\
\hline B03 & The goal of life is be devoted to Allah & $\begin{array}{c}40 \\
(2.6)\end{array}$ & $\begin{array}{c}18 \\
(1.2)\end{array}$ & $\begin{array}{c}81 \\
(5.3)\end{array}$ & $\begin{array}{c}433 \\
(28.5)\end{array}$ & $\begin{array}{c}948 \\
(62.4)\end{array}$ & 4.47 & 0.86 \\
\hline B04 & Akidah drives humans to perform prayers & $\begin{array}{c}6 \\
(0.4)\end{array}$ & $\begin{array}{c}5 \\
(0.3)\end{array}$ & $\begin{array}{c}47 \\
(3.1)\end{array}$ & $\begin{array}{c}414 \\
(27.3)\end{array}$ & $\begin{array}{l}1048 \\
(68.9)\end{array}$ & 4.64 & 0.60 \\
\hline B05 & Knowing Allah by thinking about His creation & $\begin{array}{c}32 \\
(2.1)\end{array}$ & $\begin{array}{c}23 \\
(1.5)\end{array}$ & $\begin{array}{c}74 \\
(4.90\end{array}$ & $\begin{array}{c}465 \\
(30.6)\end{array}$ & $\begin{array}{c}926 \\
(60.9)\end{array}$ & 4.47 & 0.83 \\
\hline $\mathrm{B} 06^{*}$ & Able to conceal acts from Allah knowledge & $\begin{array}{c}24 \\
(1.6)\end{array}$ & $\begin{array}{c}14 \\
(0.9)\end{array}$ & $\begin{array}{c}18 \\
(1.2)\end{array}$ & $\begin{array}{c}95 \\
(4.2)\end{array}$ & $\begin{array}{l}1369 \\
(90.1)\end{array}$ & 4.82 & 0.64 \\
\hline $\mathrm{B} 07^{*}$ & Angels cannot transform into multiple forms & $\begin{array}{c}114 \\
(7.5)\end{array}$ & $\begin{array}{c}94 \\
(6.2)\end{array}$ & $\begin{array}{l}131 \\
(8.6)\end{array}$ & $\begin{array}{c}202 \\
(12.3)\end{array}$ & $\begin{array}{c}979 \\
(64.4)\end{array}$ & 4.21 & 1.27 \\
\hline
\end{tabular}

${ }^{2}$ KBSM stands for Kurikulum Baharu Sekolah Menengah or the New Secondary School Curriculum in MAlaysia. 


\begin{tabular}{|c|c|c|c|c|c|c|c|c|}
\hline B08 & The names of angels are in the Quran & $\begin{array}{c}13 \\
(0.9)\end{array}$ & $\begin{array}{c}8 \\
(0.5)\end{array}$ & $\begin{array}{c}61 \\
(4.0)\end{array}$ & $\begin{array}{c}495 \\
(32.6)\end{array}$ & $\begin{array}{c}943 \\
(62.0)\end{array}$ & 4.55 & 0.68 \\
\hline B09 & Angels seek forgiveness for righteous person & $\begin{array}{c}45 \\
(3.0)\end{array}$ & $\begin{array}{c}43 \\
(2.8)\end{array}$ & $\begin{array}{c}95 \\
(6.2)\end{array}$ & $\begin{array}{c}462 \\
(30.4)\end{array}$ & $\begin{array}{c}875 \\
(57.6)\end{array}$ & 4.37 & 0.94 \\
\hline B10* & Angels cannot monitor human behavior & $\begin{array}{l}122 \\
(8.0)\end{array}$ & $\begin{array}{l}133 \\
(8.8)\end{array}$ & $\begin{array}{c}193 \\
(12.7)\end{array}$ & $\begin{array}{c}241 \\
(15.8)\end{array}$ & $\begin{array}{c}831 \\
(54.7)\end{array}$ & 4.00 & 1.32 \\
\hline B11 & The Prophet is a trustworthy person & $\begin{array}{c}11 \\
(0.7)\end{array}$ & $\begin{array}{c}2 \\
(0.2)\end{array}$ & $\begin{array}{c}4 \\
(0.2)\end{array}$ & $\begin{array}{c}70 \\
(4.6)\end{array}$ & $\begin{array}{l}1433 \\
(94.3)\end{array}$ & 4.92 & 0.42 \\
\hline B12* & The Prophet was not sent to all human beings & $\begin{array}{c}86 \\
(5.7)\end{array}$ & $\begin{array}{c}90 \\
(5.9)\end{array}$ & $\begin{array}{c}108 \\
(7.1)\end{array}$ & $\begin{array}{c}213 \\
(14.0)\end{array}$ & $\begin{array}{l}1023 \\
(67.3)\end{array}$ & 4.31 & 1.18 \\
\hline B13 & The Prophet was sent to perfect noble character & $\begin{array}{c}21 \\
(1.4)\end{array}$ & $\begin{array}{c}19 \\
(1.2)\end{array}$ & $\begin{array}{c}74 \\
(4.9)\end{array}$ & $\begin{array}{c}442 \\
(29.1)\end{array}$ & $\begin{array}{c}964 \\
63.4)\end{array}$ & 4.52 & 0.77 \\
\hline B14 & The Prophet had the duty to convey the teachings of Islam & $\begin{array}{c}1 \\
(0.1)\end{array}$ & $\begin{array}{c}6 \\
(0.4)\end{array}$ & $\begin{array}{c}18 \\
(1.1)\end{array}$ & $\begin{array}{c}178 \\
(11.8)\end{array}$ & $\begin{array}{l}1317 \\
86.6)\end{array}$ & 4.85 & 0.43 \\
\hline B15 & Following the lifestyle of Prophet will be rewarded & $\begin{array}{c}6 \\
(0.4)\end{array}$ & $\begin{array}{c}3 \\
(0.2)\end{array}$ & $\begin{array}{c}28 \\
(1.8)\end{array}$ & $\begin{array}{c}281 \\
(18.5)\end{array}$ & $\begin{array}{c}1202 \\
(79.1)\end{array}$ & 4.76 & 0.53 \\
\hline B16* & The prophets and messengers do not eat and drink like other humans & $\begin{array}{c}110 \\
(7.2)\end{array}$ & $\begin{array}{c}45 \\
(3.0)\end{array}$ & $\begin{array}{c}97 \\
(6.4)\end{array}$ & $\begin{array}{c}154 \\
(10.1)\end{array}$ & $\begin{array}{c}1114 \\
(73.3)\end{array}$ & 4.39 & 1.19 \\
\hline B17 & The news of the unseen is told in the scriptures revealed to the prophets & $\begin{array}{c}34 \\
(2.2)\end{array}$ & $\begin{array}{c}46 \\
(3.1)\end{array}$ & $\begin{array}{c}153 \\
(10.0)\end{array}$ & $\begin{array}{c}536 \\
(35.2)\end{array}$ & $\begin{array}{c}752 \\
(49.5)\end{array}$ & 4.27 & 0.92 \\
\hline B18 & Quran is the book of guidance for human beings & $\begin{array}{c}1 \\
(0.1)\end{array}$ & $\begin{array}{c}3 \\
(0.2)\end{array}$ & $\begin{array}{c}4 \\
(0.2)\end{array}$ & $\begin{array}{c}67 \\
(4.4)\end{array}$ & $\begin{array}{l}1445 \\
(95.1)\end{array}$ & 4.94 & 0.28 \\
\hline B19 & The teachings of Quran cultivate better character & $\begin{array}{c}3 \\
(0.2)\end{array}$ & $\begin{array}{c}3 \\
(0.2)\end{array}$ & $\begin{array}{c}4 \\
(0.3)\end{array}$ & $\begin{array}{l}145 \\
(9.5)\end{array}$ & $\begin{array}{l}1365 \\
(89.8)\end{array}$ & 4.89 & 0.38 \\
\hline B20 & Quran guides human beings towards goodness & $\begin{array}{c}2 \\
(0.1)\end{array}$ & $\begin{array}{c}3 \\
(0.2)\end{array}$ & $\begin{array}{c}6 \\
(0.4)\end{array}$ & $\begin{array}{l}111 \\
(7.3)\end{array}$ & $\begin{array}{l}1398 \\
(92.0)\end{array}$ & 4.91 & 0.34 \\
\hline B21 & The stories of the doomsday can be known through Quran & $\begin{array}{c}26 \\
(1.7)\end{array}$ & $\begin{array}{c}12 \\
(0.8)\end{array}$ & $\begin{array}{c}48 \\
(3.2)\end{array}$ & $\begin{array}{c}230 \\
(15.4)\end{array}$ & $\begin{array}{c}1201 \\
(79.0)\end{array}$ & 4.69 & 0.73 \\
\hline B22 & Dead people will raise on the doomsday & $\begin{array}{c}54 \\
(3.6)\end{array}$ & $\begin{array}{c}28 \\
(1.8)\end{array}$ & $\begin{array}{c}17 \\
(1.1)\end{array}$ & $\begin{array}{c}153 \\
(10.1)\end{array}$ & $\begin{array}{l}1268 \\
(83.4)\end{array}$ & 4.68 & 0.88 \\
\hline B23* & Humans' deeds shall not be rewarded on the doomsday & $\begin{array}{c}41 \\
(2.7)\end{array}$ & $\begin{array}{c}39 \\
(2.6)\end{array}$ & $\begin{array}{c}68 \\
(4.4)\end{array}$ & $\begin{array}{c}154 \\
(10.1)\end{array}$ & $\begin{array}{c}1218 \\
(80.1)\end{array}$ & 4.63 & 0.89 \\
\hline B24 & The human limbs and senses shall be the witnesses on the doomsday & $\begin{array}{c}23 \\
(1.5)\end{array}$ & $\begin{array}{c}8 \\
(0.5)\end{array}$ & $\begin{array}{c}18 \\
(1.2)\end{array}$ & $\begin{array}{c}185 \\
(12.1)\end{array}$ & $\begin{array}{c}1286 \\
(84.6)\end{array}$ & 4.78 & 0.64 \\
\hline B25 & The stories of the hellfire torture are extremely frightening & $\begin{array}{c}9 \\
(0.6)\end{array}$ & $\begin{array}{c}9 \\
(0.6)\end{array}$ & $\begin{array}{c}34 \\
(2.2)\end{array}$ & $\begin{array}{c}297 \\
(19.5)\end{array}$ & $\begin{array}{c}1171 \\
(77.0)\end{array}$ & 4.72 & 0.59 \\
\hline B26 & Freedom to choose between good and bad & $\begin{array}{c}153 \\
(10.1)\end{array}$ & $\begin{array}{c}81 \\
(5.3)\end{array}$ & $\begin{array}{c}225 \\
(14.8)\end{array}$ & $\begin{array}{c}509 \\
(33.4)\end{array}$ & $\begin{array}{c}552 \\
(36.3)\end{array}$ & 3.80 & 1.26 \\
\hline $\mathrm{B} 27^{\star}$ & It is unnecessary to strive to become better & $\begin{array}{c}28 \\
(1.8)\end{array}$ & $\begin{array}{c}26 \\
(1.7)\end{array}$ & $\begin{array}{l}115 \\
(7.6)\end{array}$ & $\begin{array}{c}254 \\
(16.7)\end{array}$ & $\begin{array}{l}1097 \\
(72.2)\end{array}$ & 4.56 & 0.85 \\
\hline B28 & Responsible in every act committed feelings & $\begin{array}{c}9 \\
(0.6)\end{array}$ & $\begin{array}{c}13 \\
(0.9)\end{array}$ & $\begin{array}{c}24 \\
(1.6)\end{array}$ & $\begin{array}{c}505 \\
(33.2)\end{array}$ & $\begin{array}{c}969 \\
(63.8)\end{array}$ & 4.59 & 0.63 \\
\hline B29* & Not feeling guilty in doing what is prohibited by Allah & $\begin{array}{c}23 \\
(1.5)\end{array}$ & $\begin{array}{c}17 \\
(1.1)\end{array}$ & $\begin{array}{c}55 \\
(3.6)\end{array}$ & $\begin{array}{c}187 \\
(12.3)\end{array}$ & $\begin{array}{l}1238 \\
(81.4)\end{array}$ & 4.71 & 0.73 \\
\hline B30 & Aware of one's smallness when looking at the signs of the greatness of Allah & $\begin{array}{c}41 \\
(2.7)\end{array}$ & $\begin{array}{c}16 \\
(1.1)\end{array}$ & $\begin{array}{c}40 \\
(2.6)\end{array}$ & $\begin{array}{c}279 \\
(18.4)\end{array}$ & $\begin{array}{l}1144 \\
(75.3)\end{array}$ & 4.63 & 0.82 \\
\hline B31* & Is not worried despite not performing prayers & $\begin{array}{c}33 \\
(2.2)\end{array}$ & $\begin{array}{c}68 \\
(4.5)\end{array}$ & $\begin{array}{c}129 \\
(8.5)\end{array}$ & $\begin{array}{c}288 \\
(18.9)\end{array}$ & $\begin{array}{l}1002 \\
(65.9)\end{array}$ & 4.42 & 0.97 \\
\hline B32 & Aware of the wisdom of Allah in managing this universe & $\begin{array}{c}6 \\
(0.4)\end{array}$ & $\begin{array}{c}4 \\
(0.3)\end{array}$ & $\begin{array}{c}10 \\
(0.7)\end{array}$ & $\begin{array}{c}218 \\
(14.3)\end{array}$ & $\begin{array}{c}1282 \\
(84.3)\end{array}$ & 4.82 & 0.47 \\
\hline B33 & Aware of the great power Allah in creating human beings & $\begin{array}{c}5 \\
(0.3)\end{array}$ & $\begin{array}{c}4 \\
(0.3)\end{array}$ & $\begin{array}{c}12 \\
(0.8)\end{array}$ & $\begin{array}{l}144 \\
(9.4)\end{array}$ & $\begin{array}{l}1356 \\
(89.2)\end{array}$ & 4.87 & 0.43 \\
\hline B34 & Aware that Allah always protects & $\begin{array}{c}5 \\
(0.3)\end{array}$ & $\begin{array}{c}3 \\
(0.2)\end{array}$ & $\begin{array}{c}36 \\
(2.4)\end{array}$ & $\begin{array}{c}292 \\
(19.2)\end{array}$ & $\begin{array}{l}1184 \\
(77.9)\end{array}$ & 4.74 & 0.54 \\
\hline B35 & Aware that angels pray for those who do good deeds & $\begin{array}{c}4 \\
(0.3)\end{array}$ & $\begin{array}{c}2 \\
(0.1)\end{array}$ & $\begin{array}{c}27 \\
(1.8)\end{array}$ & $\begin{array}{c}299 \\
(19.7)\end{array}$ & $\begin{array}{l}1188 \\
(78.2)\end{array}$ & 4.75 & 0.51 \\
\hline B36 & Is happy when inspired to do good deeds & $\begin{array}{c}6 \\
(0.4)\end{array}$ & $\begin{array}{c}6 \\
(0.4)\end{array}$ & $\begin{array}{c}27 \\
(1.8)\end{array}$ & $\begin{array}{c}479 \\
(31.5)\end{array}$ & $\begin{array}{l}1002 \\
(65.9)\end{array}$ & 4.62 & 0.58 \\
\hline B37* & Believe that the creation of angels is similar to the creation of human beings & $\begin{array}{c}71 \\
(4.7)\end{array}$ & $\begin{array}{l}101 \\
(6.6)\end{array}$ & $\begin{array}{c}260 \\
(17.1)\end{array}$ & $\begin{array}{c}291 \\
(19.1)\end{array}$ & $\begin{array}{c}797 \\
(52.4)\end{array}$ & 4.08 & 1.17 \\
\hline B38 & Aware that the angels Raqib dan Atid record humans activities & $\begin{array}{c}17 \\
(1.1)\end{array}$ & $\begin{array}{c}12 \\
(0.8)\end{array}$ & $\begin{array}{c}30 \\
(2.0)\end{array}$ & $\begin{array}{c}284 \\
(18.6)\end{array}$ & $\begin{array}{c}1177 \\
(77.4)\end{array}$ & 4.71 & 0.65 \\
\hline B39 & Is terrified when remembering death is near & $\begin{array}{c}16 \\
(1.1)\end{array}$ & $\begin{array}{c}22 \\
(1.4)\end{array}$ & $\begin{array}{c}56 \\
(3.7)\end{array}$ & $\begin{array}{c}387 \\
(25.4)\end{array}$ & $\begin{array}{l}1039 \\
(68.4)\end{array}$ & 4.59 & 0.73 \\
\hline B40 & Aware that the character of Prophet is the best example & $\begin{array}{c}1 \\
(0.1)\end{array}$ & $\begin{array}{c}2 \\
(0.1)\end{array}$ & $\begin{array}{c}9 \\
(0.6)\end{array}$ & $\begin{array}{c}88 \\
(5.8)\end{array}$ & $\begin{array}{l}1420 \\
(93.4)\end{array}$ & 4.92 & 0.31 \\
\hline B41 & Believe that the Prophet Muhammad went up to the sky riding Buraq & $\begin{array}{c}32 \\
(2.1)\end{array}$ & $\begin{array}{c}27 \\
(1.8)\end{array}$ & $\begin{array}{c}80 \\
(5.3)\end{array}$ & $\begin{array}{c}339 \\
(22.2)\end{array}$ & $\begin{array}{l}1042 \\
68.6\end{array}$ & 4.53 & 0.84 \\
\hline B42 & Is proud to be the people of Prophet & 3 & 5 & 5 & 90 & 1417 & 4.92 & 0.36 \\
\hline
\end{tabular}




B43 Believe that Prophet is a great preacher
B44* Is happy listening to the insult towards Prophet
B45 Aware that Quran plays role in producing successful students
B46* Aware that playing while reading Qur'an is allowed
B47 Aware that memorization of Quran facilitate the development of the brain
B48 Aware that reading Quran bring one closer to Allah
B49 The heart is calm when reading Quran
B50 Aware of the extreme awfulness of the doomsday

B51* Aware there is hell for believers

B52 Aware that the signs of doomsday have occurred

B53 Feel calm knowing believers will be safe from the grave torture

B54

B55
Has hope to receive the blessings (syafaat) from the Prophet at the field of Mahsyar

Believe there is wisdom behind every fate predetermined by Allah

Accept the provision given by Allah

Aware that Allah tests His servants so to have patience

Feel upset when what expected did not happen.

Increasing faith through voluntarily worships

Setting intention due to Allah when doing something

Striving to protect oneself from things that would tarnish the faith

Uttering the testimony of faith as a sign of remembering Allah

Reciting prayer before doing something

Hanging photos of human so that the angels enter the house

Doing good deeds in the hope the angels will write them down

Giving greeting before entering a house

Reading the stories of the angels in Qur'an

Exemplifying the trustworthiness of Prophet

Striving to exemplify the leadership characteristic of Prophet

Not reciting the phrase "peace be upon Rasulullah" when his name is mentioned

Learning and memorizing the sayings of Prophet

Pursuing the knowledge of Quran at some other places

Reading Quran in the month of Ramadan

Attending Qur'an Skills Class (KKQ) in school

Reading more entertainment magazines compared to Quran

Teaching others to read Quran $\begin{array}{lllll}(0.2) & (0.3) & (0.3) & (5.9) & (93.2)\end{array}$

$\begin{array}{ccccccc}5 & 3 & 13 & 122 & 1377 & 4.89 & 0.41 \\ (0.3) & (0.2) & (0.9) & (8.0) & (90.6) & & \end{array}$

$\begin{array}{ccccccc}8 & 15 & 23 & 43 & 1431 & 4.89 & 0.50\end{array}$

$\begin{array}{lllll}(0.5) \quad(1.0) \quad(1.5) \quad(2.8) & (94.1)\end{array}$

$\begin{array}{ccccccc}6 & 7 & 28 & 440 & 1039 & 4.64 & 0.58\end{array}$

$\begin{array}{lllll}20 & 13 & 23 & 131 & 1333\end{array}$

$\begin{array}{lllllll}(1.3) & (0.9) & (1.5) & (8.6) & (87 / 7) & 4.81 & 0.63\end{array}$

$\begin{array}{ccccccc}3 & 3 & 21 & 357 & 1136 & 4.73 & 0.51 \\ (0.2) & (0.2) & (1.4) & (23.5) & (74.7) & 4.73 & \end{array}$

$\begin{array}{lllllll}2 & 2 & 9 & 143 & 1364 & 4.88 & 0.37\end{array}$

$\begin{array}{lllllll}(0.1) & (0.1) & (0.6) & (9.4) & (89.7) & 4.88 & 0.37\end{array}$

$\begin{array}{ccccccc}2 & 4 & 6 & 183 & 1325 & 4.86 & 0.40 \\ (0.1) & (0.3) & (0.4) & (12.0) & (87.2) & 4.86 & \end{array}$

$\begin{array}{lllll}9 & 4 & 28 & 311 & 1168\end{array}$

$\begin{array}{ccccccc}(0.6) & (0.3) & (1.8) & (20.4) & (76.8) & 4.73 & 0.57 \\ 43 & 30 & 23 & 63 & 1361 & & \end{array}$

$\begin{array}{ccccccc}43 & 30 & 23 & 63 & 1361 & 4.76 & 0.82 \\ (2.8) & (2.0) & (1.5) & (4.1) & (89.5) & & \end{array}$

$\begin{array}{ccccccc}35 & 33 & 96 & 467 & 889 & 4.40 & 0.88\end{array}$

$\begin{array}{ccccccc}14 & 15 & 87 & 447 & 957 & 4.53 & 0.73 \\ (0.9) & (1.0) & (5.7) & (29.4) & (63.0) & & \end{array}$

$\begin{array}{lllll}6 & 14 & 83 & 372 & 1045\end{array}$

$\begin{array}{ccccccc}(0.4) & (0.9) & (5.5) & (24.4) & (68.8) & 4.60 & 0.67\end{array}$

$\begin{array}{ccccccc}3 & 2 & 14 & 262 & 1239 & 4.80 & 0.46\end{array}$

$\begin{array}{ccccccc}6 & 5 & 19 & 249 & 1241 & 4.79 & 0.51\end{array}$

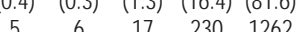

$\begin{array}{ccccccc}5 & 6 & 17 & 230 & 1262 & 4.80 & 0.49\end{array}$

$\begin{array}{lllll}125 & 420 & 460 & 242 & 273\end{array}$

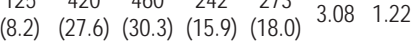

$\begin{array}{lllllll}11 & 21 & 123 & 715 & 650 & 4.30 & 0.74\end{array}$

$\begin{array}{lllllll}10.7) & (1.4) & (8.1) & (47.0) & (42.8) & 4.30 & 0.74\end{array}$

$\begin{array}{lllllll}21 & 25 & 127 & 654 & 693 & 4.30 & 0.80\end{array}$

$\begin{array}{lllll}1.4) \quad(1.6) & (8.4) & (43.0) & (45.0)\end{array}$

$\begin{array}{lllllll}25 & 12 & 57 & 508 & 918 & 4.50 & 0.76\end{array}$

$\begin{array}{lllll}1.6) & (0.8) & (3.8) & (33.4) & (60.4)\end{array}$

$\begin{array}{ccccccc}12 & 12 & 38 & 384 & 1074 & 4.64 & 0.65\end{array}$

$\begin{array}{lllll}(0.8) & (0.8) & (2.5) & (25.3) & (70.7)\end{array}$

$\begin{array}{lllllll}12 & 23 & 177 & 722 & 586 & 4.22 & 0.77\end{array}$

$\begin{array}{lllll}(0.8) & (1.5) & (11.6) & (47.5) & (38.6)\end{array}$

$\begin{array}{ccccccc}16 & 62 & 227 & 302 & 913 & 4.34 & 0.95\end{array}$

$\begin{array}{lllll}(1.1) & (4.1) & (14.9) & (19.9) & (60.1)\end{array}$

$\begin{array}{lllllll}128 & 101 & 282 & 440 & 569 & 3.80 & 1.24\end{array}$

$\begin{array}{lllll}(8.4) & (6.6) & (18.6) & (28.9) & (37.4)\end{array}$

$\begin{array}{lllllll}8 & 9 & 33 & 344 & 1126 & 4.69 & 0.60\end{array}$

$\begin{array}{llllllll}(0.5) & (0.6) & (2.2) & (22.6) & (74.1) & 4.69 & 0.60\end{array}$

$\begin{array}{lllllll}15 & 36 & 262 & 705 & 502 & 4.08 & 0.82\end{array}$

$\begin{array}{ccccccc}(1.0) & (2.4) & (17.2) & (46.4) & (33.4) & & \\ 7 & 18 & 132 & 643 & 720 & 4.35 & 0.73\end{array}$

$\begin{array}{lllllll}(0.5) & (1.2) & (8.7) & (42.3) & (47.4) & 4.35 & 0.73\end{array}$

$\begin{array}{lllll}5 & 12 & 77 & 597 & 829\end{array}$

$\begin{array}{lllllll}(0.3) & (0.8) & (5.1) & (39.3) & (54.5) & 4.47 & 0.66\end{array}$

$\begin{array}{lllllll}46 & 83 & 178 & 279 & 934 & 4.30 & 1.07\end{array}$

$\begin{array}{lllll}(3.0) \quad(5.5) & \text { (11.7) (18.4) (61.4) }\end{array}$

$\begin{array}{lllllll}52 & 107 & 456 & 616 & 289 & 3.65 & 0.98\end{array}$

$\begin{array}{llllll}(3.4) & (7.0) & (29.9) & (40.5) & (19.0)\end{array}$

$\begin{array}{lllllll}65 & 117 & 466 & 607 & 265 & 3.59 & 1.00\end{array}$

$\begin{array}{llllll}(4.3) & (7.7) & (30.7) & (39.9) & (17.4)\end{array}$

$\begin{array}{lllllll}44 & 70 & 204 & 627 & 573 & 4.07 & 0.98\end{array}$

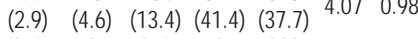

$\begin{array}{ccccccc}210 & 191 & 360 & 436 & 323 & 3.31 & 1.31 \\ (13.8) & (12.6) & (23.7) & (28.7) & (21.3) & & \end{array}$

$\begin{array}{lllll}40 & 238 & 473 & 315 & 454\end{array}$

$\begin{array}{lllllll}(2.6) & (15.7) & (31.1) & (20.7) & (29.9) & 3.60 & 1.14\end{array}$

$\begin{array}{ccccccc}105 & 176 & 344 & 576 & 319 & 3.54 & 1.15 \\ (6.9) & (11.6) & (22.6) & (37.9) & (21.0) & 3.54 & \end{array}$ 


\begin{tabular}{|c|c|c|c|c|c|c|c|c|}
\hline $\mathrm{B} 77^{*}$ & The stories of heaven and hell do not influence behavior & $\begin{array}{c}38 \\
(2.5)\end{array}$ & $\begin{array}{c}87 \\
(5.7)\end{array}$ & $\begin{array}{c}237 \\
(15.6)\end{array}$ & $\begin{array}{c}287 \\
(18.9)\end{array}$ & $\begin{array}{c}871 \\
(57.3)\end{array}$ & 4.23 & 1.06 \\
\hline B78 & Thinking about death before going to sleep & $\begin{array}{c}64 \\
(4.2)\end{array}$ & $\begin{array}{l}107 \\
(7.0)\end{array}$ & $\begin{array}{c}294 \\
(19.3)\end{array}$ & $\begin{array}{c}546 \\
(35.9)\end{array}$ & $\begin{array}{c}509 \\
(33.5)\end{array}$ & 3.87 & 1.08 \\
\hline B79 & Telling stories about the terrifying life after death & $\begin{array}{c}92 \\
(6.1)\end{array}$ & $\begin{array}{l}128 \\
(8.4)\end{array}$ & $\begin{array}{c}379 \\
(24.9)\end{array}$ & $\begin{array}{c}582 \\
(38.3)\end{array}$ & $\begin{array}{c}339 \\
(22.3)\end{array}$ & 3.62 & 1.10 \\
\hline$B 80^{*}$ & Doing acts that lead to sins & $\begin{array}{c}53 \\
(3.5) \\
\end{array}$ & $\begin{array}{c}247 \\
(16.3)\end{array}$ & $\begin{array}{c}349 \\
(23.0) \\
\end{array}$ & $\begin{array}{c}307 \\
(20.2)\end{array}$ & $\begin{array}{c}564 \\
(37.1)\end{array}$ & 3.71 & 1.22 \\
\hline B81 & Reading the stories of life after death & $\begin{array}{c}24 \\
(1.6)\end{array}$ & $\begin{array}{c}43 \\
(2.8)\end{array}$ & $\begin{array}{c}199 \\
(13.1)\end{array}$ & $\begin{array}{c}715 \\
(47.0)\end{array}$ & $\begin{array}{c}539 \\
(35.5)\end{array}$ & 4.12 & 0.85 \\
\hline B82 & Striving hard to be successful in academic & $\begin{array}{c}4 \\
(0.3)\end{array}$ & $\begin{array}{c}2 \\
(0.1)\end{array}$ & $\begin{array}{c}29 \\
(1.9)\end{array}$ & $\begin{array}{c}402 \\
(26.4)\end{array}$ & $\begin{array}{c}1083 \\
(71.3)\end{array}$ & 4.68 & 0.54 \\
\hline B83 & Relying on Allah after one's effort & $\begin{array}{c}10 \\
(0.7)\end{array}$ & $\begin{array}{c}10 \\
(0.7)\end{array}$ & $\begin{array}{c}58 \\
(3.8)\end{array}$ & $\begin{array}{c}425 \\
(28.0)\end{array}$ & $\begin{array}{l}1017 \\
(66.9)\end{array}$ & 4.60 & 0.66 \\
\hline $\mathrm{B} 84^{*}$ & Being in despair after failing in academic & $\begin{array}{c}52 \\
(3.4)\end{array}$ & $\begin{array}{c}186 \\
(12.2)\end{array}$ & $\begin{array}{c}399 \\
(26.2)\end{array}$ & $\begin{array}{c}323 \\
(21.3)\end{array}$ & $\begin{array}{c}560 \\
(36.8)\end{array}$ & 3.76 & 1.17 \\
\hline B85* & The fate and destiny of life cannot be changed & $\begin{array}{c}124 \\
(8.2)\end{array}$ & $\begin{array}{c}99 \\
(6.5)\end{array}$ & $\begin{array}{c}240 \\
(15.8)\end{array}$ & $\begin{array}{c}255 \\
(16.8)\end{array}$ & $\begin{array}{c}802 \\
(52.8)\end{array}$ & 3.99 & 1.30 \\
\hline & Overall & & & & & & 4.45 & 0.29 \\
\hline
\end{tabular}

In general, Table 3 shows the level of internalization of akidah among the respondents was high (mean $=4.45, \mathrm{sd}=$ 0.29). Meanwhile, the detailed mean score distribution analysis for three main domains and sub domains of internalization of akidah as shown above revealed two levels of mean score: high and medium high.72 internalization items obtained high mean score. Whereas 13 types of internalization obtained medium high mean score such as freedom to choose (mean $=3.80, s d=1.26$ ), feel upset when what expected did not happen (mean $=3.08, s d=1.22$ ), doing good deeds in the hope the angels would write them down (mean $=3.80, \mathrm{sd}=1.24)$, memorizing hadith (Prophetic tradition) (mean $=3.65, s d=0.98$ ), learning the Qur'an (mean $=3.59, s d=1.00$ ), attending KKQ or Qur'anic Skills Class (mean $=$ 3.31, $\mathrm{sd}=1.31$ ), love reading magazines compared to Qur'an (mean $=3.60, \mathrm{sd}=1.14$ ), teaching Qur'an (mean $=3.54$, $\mathrm{sd}=1.15$ ), thinking of death (mean $=3.87, \mathrm{sd}=1.08$ ), telling stories about the terrifying life after death (mean $=3.62, \mathrm{sd}$ $=1.10)$, committing sins (mean $=3.71, \mathrm{sd}=1.22$ ), giving up (mean $=3.76, \mathrm{sd}=1.17$ ) and believing life fate is unalterable $($ mean $=3.99$, sd $=1.30)$.

The overall analysis as shown in Table 3 revealed most of the high levels internalization of akidah among the respondents were related to the respondents' comprehension and feelings towards five out of six pillars of faith in internalizing akidah which are conviction in the existence of Allah, admitting the superiority and vast power of Allah, understanding the traits and responsibilities of the angels, understanding the roles and duties of the prophets and the knowing status of the end of the day.

Nevertheless, at the same time in internalizing akidah, the respondents' comprehension and feelings towards the beliefs in Allah's predetermined and destiny obtained a medium high level. The qada' and qadar which was classified under the comprehension domain, obtained a medium high level mean $=3.80, \mathrm{sd}=1.26$ for its 'freedom to choose' item, and in the feelings domain, its 'feel upset when what expected did not happen' item scored mean $=3.08, s d=1.22$. The frequency distribution showed $30.2 \%$ of the respondents strongly disagreed, disagreed and less agreed that humans have freedom to choose between good and bad. While the frequency distribution for 'feel upset when what expected did not happen' showed $35.8 \%$ of the respondents strongly agreed and agreed that they were frustrated over their unfulfilled expectation.

As for internalization of akidah's practice domain, the frequency distribution showed high level mean scores for some of its items such as practicing voluntarily worships (mean $=4.30, \mathrm{sd}=0.74$ ), setting intention due only to Allah $($ mean $=4.30, \mathrm{sd}=0.80)$, taking care of oneself $($ mean $=4.50, \mathrm{sd}=0.76)$, saying testimony of faith (mean $=4.64, \mathrm{sd}=$ $0.65)$, praying (mean $=4.22, \mathrm{sd}=0.77)$, hanging photos of human (mean $=4.34, \mathrm{sd}=0.95)$, saying greeting $($ mean $=$ $4.69, \mathrm{sd}=1.24)$, reading the stories of the angels (mean $=4.08, \mathrm{sd}=0.82$ ), exemplifying the trustworthiness of the Prophet (mean $=4.47, \mathrm{sd}=0.66$ ), exemplifying the leadership of the Prophet (mean $=4.47, \mathrm{sd}=0.66)$, not reciting selawat or blessing upon the prophet (mean $=4.30, \mathrm{sd}=1.07$ ), reading stories about life after death (mean $=4.12, \mathrm{sd}=$ 0.85 ), striving hard (mean $=4.68, \mathrm{sd}=0.54$ ) and relying on Allah ( mean $=4.60, \mathrm{sd}=0.66$ ).

Nevertheless, there were some items of the same domain obtained medium high mean scores namely: doing good deeds in the hope the angels would write them down (mean $=3.80, \mathrm{sd}=1.24)$, memorizing hadith (mean $=3.65, \mathrm{sd}=$ 0.98), learning Qur'an (mean $=3.59$, sd $=1.00$ ), attending KKQ class (mean $=3.31$, $s d=1.31$ ), love reading magazines compared to Qur'an (mean $=3.60$, sd $=1.14$ ), teaching Qur'an (mean $=3.54$, $s d=1.15)$, thinking of death $($ mean $=3.87$, $s d=1.08$ ), telling stories about the terrifying life after death (mean $=3.62, s d=1.10)$, committing sins (mean $=3.71$, $s d=$ 1.22), giving up (mean $=3.76, s d=1.17$ ) and believing life fate is unalterable ( mean $=3.99, \mathrm{sd}=1.30$ ). 
Meanwhile, based on frequency distribution it showed the following results; $66.3 \%$ of the respondents were doing good deeds in the hope the angels would write them down, 59.5\% of the respondents were memorizing Qur'an, 57.3\% were learning Qur'an at some other places, $50.0 \%$ were attending Qur'an skills classes in school, $50.6 \%$ of the respondents preferred reading Qur'an to magazines, 58.9\% were teaching Qur'an, $69.4 \%$ thought about death before they went to sleep, $60.6 \%$ were able to tell the terrifying stories of life after death, $57.3 \%$ were avoiding sinful acts, $58.1 \%$ did not give up after failing an examination and $69.6 \%$ believed they could alter the fate of their life.

Overall, the research outcome analysis on the level of internalization of akidah among Muslim adolescents for each concept (the foundation of akidah, faith in Allah, faith in the prophets, faith in the angels, faith in the holy scriptures and faith in qada' and qadar) showed high level mean scores. These findings were in accordance with the requisite of the teaching of Islam which places akidah at a very important position. The effort in obtaining and achieving internalization of akidah in Islam is not just limited to interest or needs but it is an ultimate instruction from Allah upon all Muslims (Wan Daud, 1989).

In this light, al-Ghazali (2005) asserted it is important to engrain the internalization of akidah at a very tender age. The process of internalizing akidah begins in a gradual and orderly manner. The effort starts with memorizing and followed by understanding the values and meaning of akidah. It is then followed by faith and conviction, and admitting the truth which is taught since childhood. Internalization of akidah among children could be obtained without the necessity of debate and proof. Nevertheless, al-Ghazali (2005) believed, the effect of internalizing akidah without proof and debate would not last long; if it is not accompanied with the strengthening and enrichment process of the children's heart.

As for that very reason, the internalization of akidah needs to go through the strengthening process such as continuous recitation of the Qur'an, comprehending the interpretation of the Qur'an, comprehending the sayings of the Prophet including the meaning and what it conveys, performing the acts of worship, attending religious classes and lectures, and associating with pious friends. Al-Ghazali's point of view was supported by a research conducted by Fatimah (1998) concerning the education of akidah among adolescents. According to Fatimah (1998), the childhood and adolescence stage are the most important stages in educating them with the best of akidah. Parents are responsible in engraining the akidah's values within their children with the help from teachers in schools. She even supported the approach proposed by al-Ghazali (n.d) in adolescence akidah education which begins with memorization, comprehension and conviction.

Nonetheless, the illustrated findings of the internalization of akidah among Muslim adolescents cannot be viewed solely on general basis. If it is viewed through a general dimension, it is plain to see that all respondents did not have any major problem in internalizing akidah. As oppose to the findings, the actual situation of our present day in reference to the sad tales of our society and problems among our adolescents; they portray a completely different circumstance. Thus, when a more detailed analysis was done on the internalization of akidah among adolescents, it is discovered that they obtained medium high scores for items such as the freedom in choosing between good and bad, being upset and in despair easily, having desire to change one's fate, learning the Qur'an, teaching the Qur'an, doing good deeds in the hope the angels will write them down, memorizing the sayings of Prophet, thinking about death, committing sins and telling terrifying stories of the doomsday.

Most of the items under the practice domain obtained medium high mean scores. One out of twenty eight items of the comprehension domain scored a medium high mean that is 'understanding qada' and qadar'. The same went for the area feelings towards faith in qada' and qadar, only one out of thirty other items in the area scored medium high score. However, under the domain practice towards akidah, eleven out of twenty seven items obtained medium high mean scores. The items can be broken down as follows: one item each from the practice towards faith in the angels and the practice towards faith in the prophets, four items from the practice towards faith in Holy Scriptures, three items from the practice towards faith in the end of the day and two items from the practice towards faith in the wills of Allah.

This research demonstrated students whom easily fall into being upset and despair were those who are average in reading and learning Qur'an, have not attempted to get acquainted with the Prophet better, fear less towards the end of the day, and were less aware in the presence of angels as the recorder of deeds. These have indirectly driven them to commit sins without the feelings of being ashamed or guilty towards anyone. The frailty in internalizing of Muslim creed was the root cause in their involvement in social delinquency such as illegal racing, black metal, drug abuse, free mingling between genders which later leads to free sex.

Thus, despite having high level of comprehension and feelings towards akidah, both did not result in high level of practice in internalizing akidah. This is aligned with the point of view of a renowned Muslim scholar, Hassan al-Banna; he pointed that the field of speech is different from the field of practice, the field of jihad is different from the field of practice, and the field of authentic jihad is different from the field of false jihad. This circumstance was also reflected upon the incident occurred among a group of the Bedouins from the Bani Asad bin Khuzaimah whom had pronounced their faith to 
the Prophet. Allah said in sura al-Hujurat, verse 14:

"The bedouins say, "We have believed." Say, "You have not [yet] believed; but say [instead], 'We have submitted,' for faith has not yet entered your hearts. And if you obey Allah and His Messenger, He will not deprive you from your deeds of anything. Indeed, Allah is Forgiving and Merciful"

According to al-Baghawi (1993), the Bedouins pronounced their faith with the intention to keep their wealth and hearts. However, when they were commanded to go on a jihad with the Muslim soldiers in the jihad campaign to Hudaibiyah, they refused to participate. In this manner, it is understood that real faith would result in one's actions in terms of practice and internalization of Allah's instructions and prohibitions.

\section{Conclusion}

In this research, there were respondents who demonstrated the consistency between their answers and the inner conflict they suffered from. For example, there was a respondent who admitted that he/she was easily upset and fall into despair. In fact, they were not even convinced that they could change for the better. The frustration that they felt even led them to choose death over living in despair. The respondent to the extent admitted that they refused to accept the predetermined fate and destiny which are against what they had wanted. Through the observation of their spiritual practice, it was discovered that the respondent was not punctual in their prayers, was still anxiety-stricken upon completing their prayers and did not practice zikir or remembrance of Allah. Furthermore, the respondents had also admitted that they were jealous towards others by not being happy looking at other people's success and happiness; to the extent they felt happy at other people's pitfalls. They also could not accept reality and was not able to control him/herself. In relation to this, the adolescents who were involved in negative activities such as illegal racing, free mingling between genders, free sex and at cetera are most probably due to their own weakness in internalizing of Muslim creed, therefore give high impact on their social aspect.

This confession could be a reflection of the present day situation faced by adolescents who are struggling with multiple challenges in their life whether within the family, friends and environmental circles or social skills. Those who are having such conflict are prone towards being influenced by negative and time-wasting activities. However, there is a probability that not all of them who are affected by such problems and immoral activities were due to the lack of religious education. In spite of that, on a general basis, the Muslim creed adolescents in Malaysia is fairly decent and within control. There are still plenty of adolescents who possess virtuous akidah and they are practicing Muslims. This is in support of the research findings which also demonstrated a high internalization among the students. What was being elaborated above took place in a minute percentage within the selected respondents; and in the overly propagated adolescence issues in the mass media. There is no denial on the involvement of adolescents in many social delinquency which to certain extent might destruct their akidah, but the number is relatively small compared to those who can still be monitored; and those whose creed and character can still be nurtured with the authentic Islamic education.

\section{References}

Abu, M. S \& Tasir, Z. 2003. Computerized data analysis: SPSS 11.5 for windows. Kuala Lumpur: Venton Publishing. al-Baghāwī, A. M. 1993. Tafsir al-Baghāwy. Beirut: Dār al-Kutb al-cilmiyah.

Bedu, H. 2008. Moral decay and social problems within family; issues and challenges. A project paper presented in Family Counseling Seminar 30 August 2008, Johor Bahru.

Bryman, A. \& Cramer, D. 1999. Quantitative data analysis with SPSS release 8 for windows: A guide for social scientists. London: Routledge.

Daradjat, Z. 1970. The roles of religion in mental health. Jakarta: Gunung Agung.

Desa, A. 2004. Stress and its coping mechanism among students. Psychology and Human Development Journal. 2004. 20: 3-12.

Fāţimah, M. K. 1998. Manhaj al-Islām fĩ tarbiyah caqīdah al-nāsyi'. Beirut: Dār al-Khayr.

al-Ghazālī , A. H. t. th. Ihya' ulum al-din. Cairo: Lubnan: Dar al-Kutub al-IImiah.

al-Ghazālī , A. H. 2005. Ihya' ulum al-din. Cairo: Dar al-Tauzic wa al-Nasyr al-Islāmiyah.

Hamka. 2003. The integration of iman and righteous practices. Shah Alam: Pustaka Dini.

Hassan, H. 2011. Satanic influence in mental phenomena in Malaysia: An analysis from Islamic belief perspective. Unpublished Ph.D. Thesis. Islamic Studies Academy: University of Malaya.

Haque, A 2001. Mental health in Malaysia issues and concerns. Kuala Lumpur. University of Malaya.

Ibrahim, S. 1996. The akidah of ahli sunah wal jamaah. Kuala Lumpur: JAKIM.

Ibrahim, Y. 2004. Social problem etiology: A research among adolescents in Sungai Petani, Kedah. Psychology and Human 
Development Journal, 20: 47-66.

Ibn Khaldūn. 2009. Mukadimah Ibn Khaldūn. Kuala Lumpur: Dewan Bahasa dan Pustaka.

Ismail, A. M. 2009. The influence of akidah in the internalization of akhlak among national secondary school students in Malaysia. Unpublished Ph.D Thesis. Faculty of Education. Bangi: The National University of Malaysia.

Langgulung, H. 1983. Mental health theories. Kajang: Pustaka Huda.

Langgulung, H. 1987. Psychology and mental health in schools. Bangi: Universiti Kebangsaan Malaysia.

Mahmood, A. 2006. The measurement of the level of internalization of Islamic education among students in national secondary schools in Malaysia. Unpublished Ph.D Thesis. Faculty of Education. Bangi: The National University of Malaysia.

Manap, J. et al. 2004. The principles of religiosity measurement and personality religiosity from the Islamic perspective. A project paper presented in Seminar on Islamic Thought, 24-27 December, The National University of Malaysia.

Kartono, K. 1974. Personality and mental hygiene theories. Bandung: Alumni.

Pallant, J. 2011. SPSS survival manual. National Library of Australia: Allen \& Unwin.

al-Qarādawī, Y. 2005. Iman and life. Shah Alam: Dewan Pustaka Fajar.

Shaleh, A. R. dan Wahab, M. A. 2004. Psychology in Islamic perspective. Jakarta: Prenada Media.

Tee, M. R. 2009. The influence of western culture in youth's life. In. Norbaya Ahmad, Haslinda Abdullah, et al. Youth development in the 21 st century. Serdang: University of Putra Malaysia.

Sābiq, S. 2003. Al-cAqa'id al-Islamiyyah. Cairo: al-Fath li al-cilm al-cAraby.

Ulwān, A. N. 1998. Tarbiyah al- Awlād. Cairo: Dār al-Salām.

Wan Daud, W. M. 1989. The concept of knowledge in Islam. New York: Mansell.

Wan Othman, W. N. 2003. Black metal: Moral decay among muslim adolescents. Unpublished Master Thesis. Faculty of Islamic Studies. Bangi: The National University of Malaysia 